\title{
Diferencias por género en una validación del FSSC-II en una muestra de estudiantes de Primaria y Secundaria
}

\author{
Federico Pulido Acosta y Francisco Herrera Clavero \\ Universidad de Granada
}

RESUMEN

Existe un enorme interés, dentro del ámbito educativo, por el campo de las emociones, ámbito que debe ser evaluado de manera adecuada. Por eso, este trabajo tiene como objetivo validar un cuestionario para evaluar el Miedo, utilizando como referencia el FSSC-II (Ascensio et al., 2012) y destinado a una muestra de alumnos de primaria (42\%) y secundaria (58\%). Para evitar que la validez del instrumento se pueda ver negativamente afectada se aplicó en análisis a ambos géneros por separado. Para esto se contó con 961 participantes, procedentes de 8 centros distintos (4 Centros de Infantil y Primaria -CEIP-y 4 Institutos de Enseñanza Secundaria -IES-). El 53,4\% fueron chicas, el $46,6 \%$ varones, el $66,3 \%$ musulmanes, el $33,7 \%$, con edades comprendidas entre los 6 y los 18 años $(M=$ 12,47). Como instrumento de evaluación se empleó el FSSC-II, adaptado al español por Ascensio et al. (2012). Para evaluar la fiabilidad se emplearon la prueba a de Cronbach junto con la prueba de dos mitades de Spearman-Brown. Posteriormente se aplicaron cálculos para comprobar la validez factorial, empleando un análisis factorial exploratorio (AFE). Los resultados obtenidos reflejan niveles de fiabilidad altos para ambos géneros. Las dimensiones esperadas fueron diferentes (6 chicos y 5 chicas), apareciendo intercorrelaciones positivas entre los totales y las dimensiones que lo conforman. Este cuestionario se muestra como instrumento adecuado para evaluar estas capacidades en la población infantojuvenil, siendo más indicado aplicar el análisis por separado.

Palabras clave: emociones, miedo, desarrollo afectivo, instrumento de evaluación, género.

\section{ABSTRACT}

In the field of education there is high interest in emotional scope. These scope must be evaluated to check this efficiently. The objective of this paper is to validate a questionnaire to assess fear, using as reference the FSSC-II (Ascensio et al., 2012) aimed at children (42\%) and teenagers (58\%). To prevent the validity be adversely affected, we applied analysis to both genders separately. We focused on 961 participants from 8 different centers ( 4 school, 4 high schools) and from 6 to 18 years old ( $M=12.47) ; 46.6 \%$ are boys and $53.4 \%$ girls; $66.3 \%$ are Muslims and $33.7 \%$ Christians. The technique used in this survey is the FSSC-Il adapted for its use in the Spanish language by Ascensio et al. (2012). To assess reliability, we used the Cronbach's a test and also the Spearman-Brown two-half test. Later, calculations were applied to verify the factorial validity, using an exploratory factor analysis (AFE). The results show high levels of reliability for both gender. The dimensions are different ( 5 girls and 6 boys) for both gender. There are positive correlations between totals and the dimensions that make them. Therefore this questionnaire is an appropriate tool to evaluate the fear in a children sample. It is more appropriate to apply the analysis separately.

Keywords: Emotions, fear, emotional development, assessment tool, gender. 


\section{Introducción}

La cambiante actualidad obliga a los sistemas educativos a retos cada vez más profundos y audaces, para adaptarse a nuevas formas culturales, sociales y políticas de manera vertiginosa, con el fin último de que el alumnado pueda aprender y desarrollar nuevas habilidades y capacidades que le permita afrontar el futuro con relativa esperanza (Codero \& Manchón, 2014). Los estados emocionales, que comienzan a adquirir una importancia cada vez mayor, van haciéndose un hueco en las más diversas áreas de la vida. Cada vez son más las voces que cuestionan la exclusividad de la inteligencia racional, como factor más influyente para alcanzar el éxito personal, social, académico y profesional, entendiendo que existen otros que también habría que tener en cuenta, como por ejemplo, el ámbito de las emociones (Filella, Pérez, Agulló, \& Oriol, 2014).

Desde esta perspectiva, se considera el concepto de emoción tomando como punto de partida las concepciones centradas en tres componentes cruciales: componente experiencial-cognitivo, componente fisiológico y reacción conductual, de carácter adaptativo. Se pone el foco en una de ellas: el miedo, considerado como emoción específica básica, destacando el componente adaptativo, que ha sido esencial para la supervivencia de la especie, destacando su función protectora a través de la reacción fisiológica que puede adoptar diferentes patrones conductuales. Otra de las características fundamentales es que se trata de una reacción negativa o de carácter desagradable y que es vivenciada como tal.
El estudio del miedo en el desarrollo infantojuvenil ha experimentado grandes avances en los últimos tiempos (García-Fernández, Inglés, Martínez-Monteagudo, Marzo, \& Estévez, 2011). Esto se debe a que los problemas de ansiedad son unos de las más prevalentes y quedan asociados con mayores alteraciones en el funcionamiento diario de las personas que los sufren, personas que se caracterizan por tener preocupaciones excesivas, persistentes y difíciles de controlar, acompañadas de conductas de hipervigilancia hacia los estímulos amenazantes y a posibles peligros. Su curso puede incluso llegar a considerarse crónico (Pardo \& Calero, 2016). Este tipo de estados puede dificultar el adecuado desarrollo del sujeto, llegando a suponer una falta de aceptación por parte de los demás, dificultades en el proceso de socialización y malestar, llegando a convertirse en problemas emocionales tales como aprensión, tensión o intranquilidad, así como depresión, desesperanza, tristeza, ambivalencia e hiperactividad, incluso pensamientos de suicidio (Gómez-Ortiz, Casas, \& Ortega-Ruiz, 2016). Estos estados emocionales pueden incidir de forma directa, sobre la propia salud, siendo un importante predictor de las determinadas enfermedades (Moreno-Rosset, Arnal-Remón, Antequera-Jurado, \& RamírezUclés, 2016).

El tratamiento de los mismos y su prevención también han conocido importantes avances gracias numerosos programas psicológicos de carácter terapéutico, centrados en el tratamiento de los problemas de ansiedad (Roca-Sánchez, Álvarez-Pérez, \& Peñate, 2014). Sin embargo, a pesar de la valía de este tipo de 
intervenciones se aboga por una intervención de carácter preventivo.

Un enfoque educativo inadecuado en el tratamiento de los miedos infantiles y adolescentes, junto con un ambiente escolar y familiar incorrectos pueden hacer que los miedos se conviertan en un fenómeno desadaptativo, incluso llegando a provocar problemas en la vida del sujeto, hasta llegar a desencadenar trastornos serios en el desarrollo. Esto es lo que puede pasar si los miedos permanecen durante un periodo largo, dando paso a una serie de alteraciones psicosomáticas.

Si se pretenden crear los enfoques y ambientes adecuados, que lleguen a evitar que este tipo de estados emocionales se conviertan en trastornos desadaptativos, será necesario el adecuado desarrollo y educación de este tipo de emociones. Por esta razón, existe un enorme interés por el desarrollo e implementación de programas de educación emocional, con independencia de la etapa educativa a la que éste vaya dirigida (Mestre, Guil, MartínezCabañas, Larrán, \& González, 2011). Para que este tipo de programas tengan éxito, deben ser evaluados de manera correcta, por lo que el estudio y evaluación del miedo en poblaciones infantiles y juveniles es un importante foco de interés en los últimos años.

Las reacciones emocionales humanas están causadas por la interacción de factores biológicos y culturales (Ford, Mauss, Troy, Smolen, \& Hankin, 2014). Los niños temen a situaciones muy variadas que, en parte, dependen de la edad. La edad es uno de los factores que influyen en la intensidad y el número de miedos, decreciendo ambos con el paso del tiempo hasta alcanzar la etapa adolescente y la adulta (Kushnir, Gothelf, \& Sadeh, 2014; Miloyan, Bulley, Pachana, \& Byrne, 2014). También se puede contemplar la enorme influencia que tiene el entorno cultural sobre el miedo, observándose la influencia este factor en el desarrollo emocional (Pulido \& Herrera, 2015; Roth et al., 2014).

Sin embargo, dentro de las variables sociodemográficas, el género es la más determinante en la influencia e intensidad de los miedos (Burnham, Hooper, \& Ogorchock, 2011; Pulido $\&$ Herrera, 2015), siendo un elemento que condiciona de manera evidente los estados emocionales. Este hecho cobra mayor relevancia en la actualidad por el significado de la dimensión psicológica que representa, algo que adquiere mayor repercusión en los últimos años en las sociedades occidentales. De esta manera, se pretende determinar con más precisión el valor predictivo $y / 0$ discriminativo dentro de esta dimensión, como reveladora de un rasgo femenino o masculino. Desde esta perspectiva Matesanz (2006), encuentra una importante objeción a los estudios es que suelen aplicar el análisis estadístico a muestras compuestas de hombres y mujeres, no por separado para ambos géneros. Esto último es teóricamente aconsejable en este tipo de estudios e incluso necesario, con la intención de obtener factores independientes para ambos géneros, factores no afectados por la naturaleza de las muestras. Sólo de esta manera se podrá considerar justamente el valor discriminativo predictivo del instrumento. La elaboración tanto de cuestionarios unidimensionales de personalidad como de inventarios multidimensionales ha 
descuidado el análisis por separado en hombres y mujeres, lo que genera dimensiones adulteradas por el factor género, constituyendo una notable fuente de error en la interpretación de las puntuaciones recogidas con estas medidas. La validez de un instrumento, característica fundamental del mismo y muy apreciada en psicometría, se puede ver negativamente afectada, cuando no se tiene en cuenta la variable género en el análisis. La estructura factorial más consistente y de mejor interpretación resulta cuando se realizan análisis factoriales separados según el género (Matesanz, 2006), por esta razón, el presenta trabajo hace especial hincapié en el análisis de los datos con independencia para ambos géneros.

Considerando todo lo mencionado, se presenta el estudio llevado a cabo con dos muestras de alumnos y alumnas escolarizados en Centros de Educación Infantil y Primaria e Institutos de Enseñanza Secundaria en la ciudad de Ceuta. Los objetivos del trabajo han sido analizar las propiedades psicométricas y validar un instrumento para evaluar el miedo considerando el FSSCII, adaptado por Ascensio et al. (2012), para una población infantojuvenil, considerando por separado a ambos géneros.

\section{Método}

\section{Participantes}

Para llevar a cabo esta investigación se seleccionó una muestra integrada por 961 participantes (513 chicas y 448 chicos) repartidos entre 8 centros educativos. Para preservar la identidad del alumnado, se emplearon nombres en clave para hacer referencia a cada uno de ellos. Considerando la etapa educativa, la muestra se dividió entre un $42 \%$ de alumnado de Primaria y el 58\% correspondiente a Secundaria. Por curso, la muestra se distribuye entre el 3,9\% de primero de Primaria, 2,2\% de segundo, $8 \%$ de tercero, $10,3 \%$ de cuarto, $8,4 \%$ de quinto y $9,3 \%$ de sexto; $9,9 \%$ de primero de ESO, $8,9 \%$ de segundo, $11,8 \%$ de alumnos de tercero y el $12,5 \%$ de cuarto. Correspondientes a los bachilleratos se alcanzan el 7,5\% y el 7,4\% para primero y segundo respectivamente. Las edades de la muestra estaban comprendidas entre los 6 y los 18 años ( $M=12,47$; D.T. = 3,316; Rango = 6-18). Describiendo la muestra, en función de la variable género, se dan porcentajes bastante equilibrados entre sí, siendo el $46,6 \%$ chicos y el resto $(53,4 \% \%)$ chicas. Considerando la cultura (que se corresponde con la religión), el grupo mayoritario es el de participantes pertenecientes a la cultura-religión musulmana. Estos constituyen el $66,3 \%$ de la muestra. El 33,7\% eran cristianos. Atendiendo al estatus, el 18,7\% de la muestra identificó su nivel como bajo. Fueron menos los que se identificaron como pertenecientes a un nivel alto $(13,1 \%)$. El 30,3\% corresponde al estatus medio-bajo y el 37,9\% al medio. Los participantes se seleccionaron por el método de muestreo no probabilístico por conveniencia, incidental o casual. Se seleccionó, aleatoriamente, un curso (desde primero a sexto de Primaria) por cada uno de los centros. El error muestral fue del $3 \%$.

\section{Instrumento}

La intención fue la utilizar un instrumento que permitiera evaluar el miedo a través de una escala preestablecida. Para ello se empleó 
Tabla 1

Variables sociodemográficas de la muestra

\begin{tabular}{|c|c|c|c|c|}
\hline \multicolumn{5}{|c|}{ Variables sociodemográficas } \\
\hline & $N$ & $M$ & $D T$ & Rango \\
\hline \multirow[t]{2}{*}{ Edad } & 961 & 12,47 & 3,316 & $6-18$ \\
\hline & & $\mathrm{N}$ & $\%$ & \\
\hline \multirow[t]{2}{*}{ Etapa educativa } & Primaria & 404 & 42,0 & \\
\hline & Secundaria & 557 & 58,0 & \\
\hline \multirow{2}{*}{ Sexo } & Varones & 448 & 46,6 & \\
\hline & Mujeres & 513 & 53,4 & \\
\hline \multirow[t]{2}{*}{ Cultura/religión } & Cristianos & 324 & 33,7 & \\
\hline & Musulmanes & 637 & 66,3 & \\
\hline \multirow[t]{5}{*}{ Estatus } & Bajo & 180 & 18,7 & \\
\hline & Medio-bajo & 291 & 30,3 & \\
\hline & Medio & 364 & 37,9 & \\
\hline & Alto & 126 & 13,1 & \\
\hline & Mujeres & 47 & 3,7 & \\
\hline
\end{tabular}

la traducción del Inventario de Miedo para Niños (FSSC-II), adaptada para su uso en español por Ascensio et al. (2012). En el cuestionario se pide que el sujeto conteste, empleando una escala tipo Likert, la cantidad de miedo (desde nada hasta mucho) que le producen un conjunto de estímulos que se pueden agrupar dentro de diferentes categorías. Sin embargo, el mencionado instrumento fue validado en una muestra de alumnos adolescentes en el contexto mexicano. En este caso, pretende validarse esta misma versión en una muestra de alumnos de edades similares, pero analizando por separado ambos géneros, diferente al del cuestionario de referencia. Dicho instrumento parte de una visión integradora del miedo, presentándolo como una emoción que se produce ante un grupo de estímulos determinados, propios de la edad infantojuvenil. El instrumento pretende medir un área unitaria (miedo), que a su vez se divide en diferentes tipos (los factores del cuestionario original fueron 5). Para llevar a cabo la validación y el análisis psicométrico del instrumento se contó con la opinión de dos expertos en los campos de la psicología, las emociones, la educación, la evaluación y la estadística.

Con respecto a las diferentes dimensiones que aparecen en el cuestionario de referencia, se muestran factores centrados en la manifestación de miedo relacionado con la muerte y elementos referentes a peligros físicos que pueden conducir a ella (miedo a la muerte y al peligro). Aparecen también ítems próximos a la manifestación de miedo ante estímulos relacionados con animales y lesiones físicas (miedo a los animales o a las lesiones), con situaciones estresantes provocadas por elementos escolares y sociales (miedo al fracaso escolar o a la crítica) así como relacionada con elementos 
extraños (miedo a lo desconocido). Finalmente aparece una dimensión cercana a los miedos provocados por acontecimientos próximos a situaciones sanitarias (miedos médicos). El número total de ítems en el cuestionario original es de 78 distribuido entre los diferentes factores. Para el sistema de respuestas se usa la ya mencionada escala tipo Likert.

\section{Procedimiento}

Tras solicitar y obtener las pertinentes autorizaciones en la Dirección Provincial de Educación, se habló con el equipo directivo de los centros que voluntariamente participaron en esta experiencia. Una vez hecho este paso inicial, se redactaron los documentos para recoger por escrito el consentimiento de los padres de los menores que constituirían la muestra (la mayor parte de la misma). En cada uno de los diferentes centros, se pasaron los cuestionarios. Se respetó escrupulosamente la confidencialidad, contestando al cuestionario los alumnos que quisieron hacerlo de manera voluntaria. Antes de contestar los cuestionarios, se explicó el modo en el que debían contestar al mismo. Después de esto, los alumnos contestaron por sí mismos. Se intentó reducir al máximo la influencia de elementos externos que pudieran dificultar la concentración del alumnado, dentro de cada una de las clases correspondientes, configurando un entorno tranquilo y libre de distracciones en función de sus posibilidades. Finalmente, antes de la entrega, se aseguró que ninguno de los participantes dejara ítems sin contestar. La duración de la prueba fue de unos 35 minutos máximo.

Una vez obtenidos los datos y construida la base, se llevó a cabo el pertinente análisis estadístico. Antes de iniciar el análisis del cuestionario se procedió a realizar una primera valoración para contemplar la existencia de diferencias significativas en los totales de miedo empleando como variable independiente. Para ello se empleó la prueba ANOVA. Una vez evaluadas estas diferencias se continuó con el análisis de la consistencia interna del cuestionario. Para evaluar la fiabilidad se emplearon la prueba a de Cronbach junto con la prueba de dos mitades de Spearman-Brown. Posteriormente se aplicaron cálculos para comprobar la validez factorial, empleando un análisis factorial exploratorio (AFE), por medio del análisis de varianza de componentes principales con rotación varimax. La fiabilidad para cada una de las muestras se comprobó tanto para el cuestionario, como para cada uno de los factores (5-6) obtenidos en cada caso. Para asegurar la validez del modelo jerárquico propuesto, se aplicaron cálculos correlacionales entre el cuestionario (miedo) y cada uno de los factores del mismo. Finalmente se volvió a usar la prueba ANOVA para contemplar la existencia de diferencias significativas en los factores de miedo que coinciden en ambos géneros, al mismo tiempo que se contemplaron las medias de cada factor, para comprobar dónde se dan los niveles más altos y más bajos para ambos géneros.

\section{Resultados}

Se comienza con los resultados que hacen referencia a la existencia de diferencias significativas para ambos géneros. Los resultados de la prueba ANOVA resaltan la existencia de 
diferencias significativas en los niveles totales de miedo total ( $p<0,001)$. Las niñas reflejan un nivel más alto de miedo que los niños, tal y como demuestran las medias $(126,26$ las chicas y 95,92 los varones).

Tras contemplar la existencia de diferencias se separaron ambas muestras y se procedió a realizar el análisis de la consistencia interna del instrumento para cada una de ellas. La fiabilidad para la medida del miedo, a través del cuestionario empleado se evaluó, en primer caso, a través de la prueba de Cronbach. Así, la consistencia interna del cuestionario (miedo) fue de 0,955, para las chicas. Para los chicos el resultado obtenido fue de 0,961. Se observa un resultado muy similar en cuanto a la fiabilidad del cuestionario, independientemente del género. Del mismo modo, ambos son prácticamente idénticos a los obtenidos en el cuestionario de referencia. En un segundo análisis se procedió a realizar la prueba de dos mitades de Spearman-Brown. Este coeficiente fue de 0,934 para el cuestionario al que contestaron los chicos, mientras que para las chicas fue de 0,912 . Se observa una gran igualdad para ambos géneros incluyendo todos los ítems.

Los factores encontrados en el género femenino fueron 5 (Tabla 2). Para el primer factor, miedo a la muerte y al daño, el valor del de Cronbach fue de 0,918 . El del segundo factor, miedo a lo desconocido y a los médicos fue de 0,863 . Con el tercer factor, que hace referencia al miedo relacionado con elementos escolares y la crítica (miedo a la crítica y la escuela) se obtuvo 0,856. Para el factor miedo a los animales fue de 0,851. Para el último factor, el miedo a la soledad fue de 0,716 . Los resultados del se- gundo análisis (Spearman-Brown) para los factores fue de 0,$913 ; 0,836 ; 0,837 ; 0,799$ y 0,694 respectivamente (siguiendo el orden en el que se han comentado anteriormente). Los valores de la primera prueba (a de Cronbach) quedan recogidos en la Tabla 3.

En el caso de los varones, los factores fueron 6 (Tabla 4): en miedo a lo desconocido y los animales, cuyo valor del de Cronbach fue de 0,934 . El del segundo, miedo a la muerte y al daño fue de 0,875 . Con tercer factor, que hace referencia al miedo provocado por padres y familiares se obtuvo 0,826. Para el cuarto factor, miedo al peligro, fue de 0,839. El penúltimo, relacionado con miedos provocados por diferentes fenómenos escolares (miedos escolares) dio 0,742 . Para el factor final, relacionado con el miedo ante la crítica social fue de 0,700. En el segundo análisis, este coeficiente fue de 0,921; 0,$857 ; 0,830 ; 0,783 ; 0,779$ y 0,630 respectivamente (siguiendo el mismo orden). Los valores de la primera prueba (a de Cronbach) quedan recogidos en la Tabla 5.

En el apartado relacionado con la varianza factorial se emplearon diferentes análisis factoriales exploratorios (AFE). En este sentido, los factores obtenidos en los varones, como se ha mencionado, fueron 6 . El primero, se vinculó con el miedo a lo desconocido y los animales, con un total de 28 ítems, que representaban el $27,120 \%$ de la varianza explicada. Los reactivos que configuran esta categoría se dividen en dos apartados. Por un lado aparecen ítems que se relacionan con los miedos provocados por elementos desconocidos (como Las casas misteriosas, La oscuridad o La gente desconocida). Por otro lado, se encuentran ítems 
Tabla 2

Ponderaciones de factores para el análisis factorial exploratorio con rotación varimax de la FSSC-Il en mujeres

\begin{tabular}{|c|c|c|c|c|c|}
\hline ÍTEMS & Muerte & $\begin{array}{l}\text { Desconocido/ } \\
\text { Médicos }\end{array}$ & $\begin{array}{l}\text { Crítical } \\
\text { Escuela }\end{array}$ & Animales & Soledad \\
\hline 51 Ladrón &. .623 & .099 & .235 & .076 & .048 \\
\hline 22 Secuestro & .580 & -.078 & .138 & .023 & .091 \\
\hline 36 Arma & .601 & .057 & .167 & .042 & .056 \\
\hline 19 Asesinos & .600 & .004 & .081 & .174 & .105 \\
\hline 13 Guerra nuclear & .646 & -.018 & .064 & .079 & .110 \\
\hline 33 Atropello & .558 & .075 & .220 & .058 & -.020 \\
\hline 56 Terremotos & .640 & .063 & .145 & .281 & .018 \\
\hline 66 Tigres & .698 & .304 & .033 & .431 & -.049 \\
\hline 38 Asfixia & .426 & -.013 & .205 & .041 & .022 \\
\hline 78 Tiburones & .569 & .064 & .143 & .345 & -.122 \\
\hline 44 Electricidad & .538 & .154 & .254 & .119 & -.051 \\
\hline 15 Drogas & .587 & -.176 & .208 & .013 & .050 \\
\hline 68 Lugar extraña & .566 & .417 & .157 & .059 & .120 \\
\hline 76 Alturas & .568 & .234 & .134 & .112 & .045 \\
\hline 11 Invasión país & .572 & .081 & .137 & .061 & .227 \\
\hline 46 Perderse & .572 & .436 & .194 & -.052 & .179 \\
\hline 25 Fuego & .586 & .186 & .055 & .368 & .132 \\
\hline 37 Incendios & .497 & .283 & .096 & .110 & .154 \\
\hline 42 Serpientes & .573 & .284 & -.003 & .492 & .010 \\
\hline 32 Muerte personal & .559 & .323 & .151 & -.140 & -.052 \\
\hline 31 Ciclones & .519 & .128 & .119 & .227 & .099 \\
\hline 45 Accidente familia & .336 & -.020 & .287 & .038 & .025 \\
\hline 2 Malabares & .493 & .010 & -.090 & .090 & .282 \\
\hline 67 Muertos & .498 & .492 & .175 & .185 & -.063 \\
\hline 26 Operación & .472 & .214 & .197 & .233 & -.008 \\
\hline 21 Pelea & .465 & .241 & .242 & .026 & .406 \\
\hline 64 Sida & .386 & -.157 & .179 & .167 & .042 \\
\hline 60 Acoso & .424 & .058 & .323 & .098 & .185 \\
\hline 23 Enfermedad seria & .282 & -.055 & .196 & .078 & .049 \\
\hline 17 Violencia televisión & .310 & .192 & .210 & .149 & .248 \\
\hline 72 Sangre & .216 & .676 & .053 & .121 & .049 \\
\hline 9 Doctor & .587 & .548 & .057 & .132 & -.021 \\
\hline 74 Desconocidos & .154 & .613 & .107 & -.073 & .087 \\
\hline 53 Solo casa & .566 & .636 & .071 & -.022 & .193 \\
\hline 57 Inyección & -.176 & .641 & .119 & .224 & -.061 \\
\hline 49 Gente extraña & .568 & .572 & .116 & -.044 & .116 \\
\hline 77 Hospitalización & .417 & .646 & .198 & .059 & -.083 \\
\hline 52 Pesadillas & .572 & .617 & .117 & .148 & .354 \\
\hline 27 Escuela & .234 & .497 & .050 & .034 & .016 \\
\hline 24 Conocer alguien & .572 & .537 & .136 & .068 & .240 \\
\hline 69 Tormentas & .081 & .601 & .075 & .312 & .148 \\
\hline 14 Dentista & .586 & .576 & .015 & .307 & .072 \\
\hline 41 Borrachos & .436 & .577 & .103 & .049 & .089 \\
\hline 75 Avión & .497 & .592 & -.030 & .068 & $\begin{array}{l}-.072 \\
\text { (Contin }\end{array}$ \\
\hline
\end{tabular}


Tabla 2

Ponderaciones de factores para el análisis factorial exploratorio con rotación varimax de la FSSC-Il en mujeres (continuación)

\begin{tabular}{|c|c|c|c|c|c|}
\hline 16 Salón & -.158 & .433 & .060 & .045 & .094 \\
\hline 39 Castigo & .193 & .513 & .477 & .053 & .004 \\
\hline 65 Casas Misteriosas & .327 & .517 & .114 & .170 & .289 \\
\hline 4 Autobús & -.100 & .298 & .046 & .040 & .061 \\
\hline 71 Perros & .254 & .506 & .126 & .489 & .118 \\
\hline 70 Cementerios & .408 & .497 & .029 & .238 & -.043 \\
\hline 35 Fantasmas & .193 & .513 & .108 & .304 & .498 \\
\hline 28 Familiar muerte & .278 & .322 & .227 & .104 & .047 \\
\hline 55 Colegio nuevo & .017 & .388 & .286 & .061 & .132 \\
\hline 79 Suspender Examen & .327 & .220 & .657 & .078 & -.043 \\
\hline 40 Suspender & .364 & .060 & .570 & .072 & .014 \\
\hline 59 Examen & -.066 & .245 & .596 & .280 & -.095 \\
\hline 10 Malas notas & .317 & -.190 & .533 & .141 & .121 \\
\hline 61 Notas escolares & -.054 & .297 & .570 & .215 & -.068 \\
\hline 50 Castigo padre & .231 & .493 & .557 & .083 & .005 \\
\hline 30 Discusión padres & .340 & .027 & .556 & .038 & .108 \\
\hline 29 Errar & .078 & .260 & .501 & .089 & .303 \\
\hline 47 No amigos & .284 & .141 & .543 & -.121 & .036 \\
\hline 20 Padres critiquen & .220 & .307 & .508 & .039 & .356 \\
\hline 7 Perder amigos & .215 & .003 & .432 & -.039 & .182 \\
\hline 43 Divorcio padres & .431 & .062 & .449 & -.016 & -.075 \\
\hline 34 Director & .435 & .428 & .450 & -.165 & .096 \\
\hline 48 Enfermedad familiar & .312 & .031 & .349 & -.002 & .009 \\
\hline 73 Tonto & .283 & .272 & .414 & .006 & .120 \\
\hline 6 Ratones & .324 & .017 & .068 & .913 & .252 \\
\hline 54 Ratas & .434 & .235 & -.003 & .886 & -.008 \\
\hline 63 Lagartijas & .210 & .359 & .059 & .743 & -.014 \\
\hline 18 Arañas & .178 & .140 & .133 & .723 & .164 \\
\hline 58 Abejas & .299 & .349 & .129 & .484 & -.270 \\
\hline 62 Truenos & .205 & .397 & .147 & .407 & .216 \\
\hline 3 Soledad & .107 & .366 & .017 & .016 & .564 \\
\hline 12 Oscuridad & .115 & .540 & -.117 & .167 & .574 \\
\hline 5 Críticas & .140 & .148 & .273 & -.090 & .409 \\
\hline 8 Cerrado & .318 & .367 & .061 & .174 & .460 \\
\hline 1 Engaño & -.033 & -.187 & .253 & .242 & .344 \\
\hline
\end{tabular}


Tabla 3

Valores de la prueba a de Cronbach en cuestionario y factores de chicas

\begin{tabular}{lll}
\hline \multicolumn{1}{c}{ Miedo total } & a de Cronbach & 0,955 \\
\hline \multicolumn{1}{c}{ Factores } & a de Cronbach \\
\hline Miedo muerte/daño & 0,918 \\
Miedos desconocido/médico & 0,863 \\
Miedo crítica/escuela & 0,856 \\
Miedo animales & 0,851 \\
Miedos soledad & 0,694 \\
\hline
\end{tabular}

Tabla 4

Ponderaciones de factores para el análisis factorial exploratorio con rotación Varimax de la FSSC-Il en varones

\begin{tabular}{|c|c|c|c|c|c|c|}
\hline Ítems & $\begin{array}{l}\text { Desconocido/ } \\
\text { Animales }\end{array}$ & Muerte & Padres & Peligro & Escolares & Crítica social \\
\hline 53 Solo casa & .645 & .095 & .147 & .035 & -.114 & .046 \\
\hline 16 Salón & .541 & -.074 & .047 & -.161 & .082 & -.121 \\
\hline 65 Casas Misteriosas & .686 & .153 & .072 & .261 & -.022 & -.028 \\
\hline 41 Borrachos & .700 & .210 & .236 & .126 & .156 & .042 \\
\hline 69 Tormentas & .713 & .108 & .086 & .336 & .074 & -.064 \\
\hline 35 Fantasmas & .727 & .195 & .126 & .178 & -.134 & -.160 \\
\hline 74 Desconocidos & .610 & .069 & .199 & .254 & .087 & .049 \\
\hline 49 Gente extraña & .645 & .135 & .236 & .141 & -.010 & .005 \\
\hline 72 Sangre & .639 & .117 & .162 & .202 & .026 & .106 \\
\hline 63 Lagartijas & .539 & -.007 & -.015 & .103 & .081 & .022 \\
\hline 52 Pesadillas & .658 & .173 & .250 & .118 & -.078 & -.019 \\
\hline 14 Dentista & .619 & .101 & .053 & -.082 & .148 & -.062 \\
\hline 9 Doctor & .518 & -.042 & -.042 & -.091 & .178 & -.026 \\
\hline 12 Oscuridad & .613 & .082 & .069 & -.034 & -.125 & .027 \\
\hline 75 Avión & .617 & .062 & .025 & .159 & .103 & .010 \\
\hline 71 Perros & .608 & .077 & .094 & .249 & .106 & .021 \\
\hline 54 Ratas & .528 & .179 & .001 & .281 & .132 & .140 \\
\hline 18 Arañas & .524 & .141 & -.057 & .052 & .059 & .170 \\
\hline 24 Conocer alguien & .550 & -.011 & -.001 & -.090 & .321 & -.001 \\
\hline 57 Inyección & .572 & .187 & .130 & .117 & .126 & -.122 \\
\hline 42 Serpientes & .593 & .317 & .112 & .367 & -.075 & .044 \\
\hline 70 Cementerios & .570 & .210 & .109 & .317 & -.031 & .017 \\
\hline 4 Autobús & .436 & -.240 & -.030 & -.075 & .068 & .012 \\
\hline 8 Cerrado & .540 & .159 & .115 & .156 & -.180 & .219 \\
\hline 21 Pelea & .557 & .243 & .135 & .170 & .109 & .222 \\
\hline 77 Hospitalización & .535 & .251 & .118 & .146 & .195 & -.048 \\
\hline 46 Perderse & .552 & .317 & .341 & .234 & -.085 & -.057 \\
\hline 17 Violencia televisión & .459 & -.007 & .141 & .095 & .125 & .232 \\
\hline 6 Ratones & .397 & .125 & -.035 & .128 & .006 & .299 \\
\hline 62 Truenos & .424 & .028 & -.011 & .376 & .150 & .021 \\
\hline 3 Soledad & .442 & .068 & .094 & -.032 & -.138 & .204 \\
\hline 58 Abejas & .426 & .115 & -.108 & .261 & .144 & .222 \\
\hline
\end{tabular}


Tabla 4

Ponderaciones de factores para el análisis factorial exploratorio con rotación Varimax de la FSSC-Il en varones (continuación)

\begin{tabular}{|c|c|c|c|c|c|c|}
\hline 37 Incendios & .497 & .337 & .283 & .210 & .081 & .027 \\
\hline 55 Colegio nuevo & .400 & -.012 & .080 & .146 & .211 & .206 \\
\hline 27 Escuela & .419 & -.236 & -.031 & -.021 & .171 & .057 \\
\hline 22 Secuestro & .112 & .800 & .202 & .124 & .026 & .119 \\
\hline 13 Guerra nuclear & .004 & .873 & -.057 & .085 & .116 & .237 \\
\hline 33 Atropello & .144 & .762 & .296 & .166 & .042 & -.197 \\
\hline 36 Arma & .177 & .726 & .137 & .326 & .024 & .016 \\
\hline 11 Invasión país & .175 & .730 & .092 & -.072 & -.041 & .327 \\
\hline 32 Muerte personal & .195 & .744 & .261 & .112 & .013 & -.210 \\
\hline 38 Asfixia & .100 & .522 & .303 & .070 & .062 & -.025 \\
\hline 31 Ciclones & .298 & .676 & .167 & .221 & .066 & -.051 \\
\hline 51 Ladrón & .316 & .607 & .272 & .224 & -.052 & .093 \\
\hline 26 Operación & .218 & .633 & .153 & .248 & .100 & -.020 \\
\hline 23 Enfermedad seria & .025 & .538 & .334 & .001 & .043 & .117 \\
\hline 19 Asesinos & .214 & .601 & .043 & .374 & .057 & .236 \\
\hline 44 Electricidad & .263 & .554 & .350 & .316 & -.050 & .000 \\
\hline 15 Drogas & -.188 & .595 & .091 & .241 & .107 & .250 \\
\hline 28 Familiar muerte & -.274 & .379 & .362 & .166 & -.093 & .181 \\
\hline 67 Muertos & .387 & .463 & .257 & .382 & .082 & -.126 \\
\hline 64 Sida & -.194 & .353 & .188 & .300 & .118 & -.008 \\
\hline 60 Acoso & .130 & .337 & .289 & .266 & .139 & .249 \\
\hline 30 Discusión padres & .074 & .221 & .697 & .124 & .008 & .136 \\
\hline 43 Divorcio padres & .016 & .293 & .728 & .091 & -.051 & -.044 \\
\hline 40 Suspender & .038 & .246 & .636 & .094 & .392 & .003 \\
\hline 50 Castigo padre & .315 & .108 & .654 & .111 & .318 & -.034 \\
\hline 47 No amigos & .128 & .137 & .697 & -.009 & -.222 & .226 \\
\hline 79 Suspender Examen & .110 & .197 & .616 & .088 & .492 & .024 \\
\hline 39 Castigo & .387 & .147 & .576 & .066 & .258 & -.069 \\
\hline 73 Tonto & .215 & .005 & .600 & .257 & .101 & .197 \\
\hline 48 Enfermedad familiar & .023 & .264 & .467 & .094 & -.002 & .114 \\
\hline 45 Accidente familia & -.028 & .456 & .422 & .084 & -.084 & .082 \\
\hline 34 Director & .382 & .361 & .524 & .148 & .159 & .009 \\
\hline 20 Padres critiquen & .345 & .142 & .523 & -.070 & .046 & .246 \\
\hline 29 Errar & .349 & .193 & .443 & .005 & .270 & .158 \\
\hline 78 Tiburones & .131 & .254 & .079 & .803 & .039 & .083 \\
\hline 66 Tigres & .281 & .228 & .109 & .760 & .044 & .033 \\
\hline 56 Terremotos & .148 & .452 & .170 & .659 & .043 & .061 \\
\hline 76 Alturas & .143 & .342 & .192 & .606 & .013 & .063 \\
\hline 68 Lugar extraña & .462 & .321 & .246 & .545 & .007 & -.034 \\
\hline 25 Fuego & .248 & .219 & .116 & .481 & .040 & .167 \\
\hline 61 Notas escolares & .251 & .072 & .113 & .020 & .790 & .018 \\
\hline 59 Examen & .265 & .079 & .045 & .173 & .579 & .181 \\
\hline 7 Perder amigos & -.054 & .213 & .411 & .020 & -.104 & .606 \\
\hline 5 Críticas & .223 & .036 & .152 & .042 & -.008 & .402 \\
\hline 2 Malabares & .233 & .078 & .025 & .213 & .035 & .440 \\
\hline 10 Malas notas & -.212 & .315 & .379 & .121 & .289 & .472 \\
\hline 1 Engaño & -.056 & -.001 & .062 & -.010 & .061 & .315 \\
\hline
\end{tabular}


Tabla 5

Valores de la prueba a de Cronbach en cuestionario y factores en chicos

\begin{tabular}{|c|c|c|}
\hline Miedo total & a de Cronbach & 0,961 \\
\hline Factores & a de Cronbach & \\
\hline Miedo desconocido/animal & 0,934 & \\
\hline Miedo muerte/daño & 0,875 & \\
\hline Miedos padres y familiares & 0,826 & \\
\hline Miedo peligro & 0,783 & \\
\hline Miedos escolares & 0,779 & \\
\hline Miedo crítica social & 0,630 & \\
\hline
\end{tabular}

relacionados con animales de diferente tipo (Los perros, Las lagartijas, Las ratas, Las arañas o Los ratones). El segundo de los factores está conformado por 13 ítems, que representan el $8,345 \%$ de la varianza explicada. Este hace referencia a los miedos relacionados con la muerte y el daño (miedo a la muerte y el daño). Dentro de este factor aparecen ítems como No poder respirar, Tener una enfermedad seria - Contraer el SIDA relacionados con la muerte. Recibir una descarga eléctrica o Que me operen están relacionados con el daño personal. El ter- cero se relaciona con miedos provocados por eventos cercanos a los padres y familiares. Son 10 los ítems que la conforman, entre los que se incluyen Que alguien de mi familia se ponga enfermo, Que mis padres se estén divorciando, o Que alguien de mi familia tenga un accidente. Esta categoría representa el 5,83\% de la varianza explicada. El cuarto factor también está conformado por 10 ítems, cercanos a diferentes tipos de peligros (miedo al peligro), como por ejemplo Los terremotos, Caerme de lugares altos, El fuego o Las tormentas. Esta categoría

Tabla 6

Varianza e ítems de la agrupación por factores en el cuestionario para chicos

\begin{tabular}{lll}
\hline Factor & No ítems & Varianza explicada \\
\hline \multirow{2}{*}{ Miedo desconocido/animales } & 28 & $27,120 \%$ \\
$\stackrel{\frac{0}{2}}{\Sigma}$ Miedo muerte/daño & 13 & $8,345 \%$ \\
Miedo padres y familiares & 10 & $5,831 \%$ \\
Miedos escolares & 10 & $2,534 \%$ \\
Miedo crítica social & 8 & $2,425 \%$ \\
Total & 9 & $2,053 \%$ \\
\hline
\end{tabular}


representa el 2,534\% de la varianza explicada. El quinto factor, relacionado con los miedos escolares está constituido por 8 ítems, que representan el $2,425 \%$ de la varianza explicada. Dentro de este factor destacan los ítems en los que se indica que la participante conteste cuanto miedo le producen elementos que se relacionan con Suspender un examen, Sacar malas notas o Estar esperando para hacer un examen. El miedo a la crítica social encierra 9 ítems, como Cometer errores, Perder a mis amigos o Que otros me critiquen, que representa el 2,053\% de la varianza explicada. Entre todos los factores suman una varianza explicada total del $48.308 \%$, con un total de 78 reactivos. Todos los resultados en este apartado aparecen resumidos en la Tabla 6 .

En este apartado, como señaló, los factores obtenidos entre las chicas fueron 5. El primero de los factores está conformado por 26 ítems, que representan el $25,882 \%$ de la varianza explicada. Este hace referencia a los miedos relacionados con la muerte y el daño (miedo a la muerte y el daño). Dentro de este factor aparecen ítems como No poder respirar, Que me atro- pelle un camión, Tener una enfermedad seria o Contraer el SIDA relacionados con la muerte. Recibir una descarga eléctrica o Que me operen están relacionados con el daño personal. Es la categoría que más relación tiene con los factores descritos en la población masculina, pudiéndose equiparar ambas. El segundo factor está relacionado con lo desconocido y los médicos. En este factor, que engloba un total de 18 ítems, se incluyen ítems relacionados con elementos desconocidos (Las casas misteriosas, La oscuridad o La gente desconocida), sin embargo también integra ítems relacionados con situaciones sanitarias (Que me pongan una inyección, Ir al doctor o Tener que ir al hospital), por lo que no se puede equiparar con el factor descrito en los varones. Representan el 8,92\% de la varianza explicada. Dentro del tercer factor se mencionan los miedos provocados por diferentes situaciones escolares (miedos escolares) con ítems como Suspender, Sacar malas notas o Cuando me dan las notas en el instituto/ colegio. Se podrían equiparar si no se incluyeran también ítems relacionados con la crítica social (quedando este factor dividido en dos

Tabla 7

Varianza e ítems de la agrupación por factores en el cuestionario para chicas

\begin{tabular}{lll}
\hline Factor & No ítems & Varianza explicada \\
\hline Miedo muerte/daño & 26 & $25,882 \%$ \\
$\stackrel{\circ}{\circ}$ Miedo desconocido/médicos & 18 & $8,920 \%$ \\
$\stackrel{\varrho}{\sum}$ Miedos escolares/crítica & 15 & $6,656 \%$ \\
Miedo animales & 10 & $2,970 \%$ \\
Miedo soledad & 9 & $2,401 \%$ \\
Total & 78 & $46,829 \%$ \\
\hline
\end{tabular}


en los varones), como Que mis padres me critiquen o Perder a mis amigos. Los ítems que lo conforman son 15, con una varianza explicada de 6,656\%. El miedo a los animales es otra categoría que no se puede equiparar (en los varones incluye elementos relacionado con lo desconocido), estando constituida por 10 ítems, que representan el $2,970 \%$ de la varianza explicada. Dentro de este factor destacan los ítems en los que se indica al alumno que conteste cuanto miedo le producen animales como Los ratones, Las ratas, Las arañas, o Las lagartijas. El factor final encontrado entre las chicas, está relacionado con miedos provocados por la soledad (miedo a la soledad). Encierra 9 ítems, como Estar solo, La oscuridad o Estar en lugares cerrados, que representa el 2,401\% de la varianza explicada. Entre todos los factores suman una varianza explicada total del $45,740 \%$, con un total de 78 reactivos. Todo aparece en la Tabla 7.

De la misma manera que hace el cuestionario de referencia, se pretende medir un área unitaria, el miedo, a su vez subdivido en una serie de temores ante estímulos específicos. Para este planteamiento jerárquico es necesario que las diferentes puntuaciones correlacionen positivamente entre sí. Todas las correlaciones, en el caso de las varones, fueron positivas y significativas al nivel $p=0,01$, tal y como era de esperar (Tabla 8). Las correlaciones más bajas entre los totales y los factores se dan entre el miedo total y el factor de miedos escolares $(0,692)$, mientras que las más altas $(0,882)$ se dan entre los totales y el factor de miedo a la muerte y al daño. Por otro lado, los mismos resultados comentados para los totales y todas las categorías se repiten considerando las intercorrelaciones de los factores entre sí. En todos los casos el nivel de significación es de al menos $p=0,01$, repitiéndose los resultados del anterior apartado. En este sentido, los niveles son más bajos que los reflejados para los totales (algo esperado), moviéndose entre los más altos $(0,771)$ encontrados en las correlaciones entre el miedo a la muerte y al daño y el miedo

Tabla 8

Correlaciones entre los factores en chicos

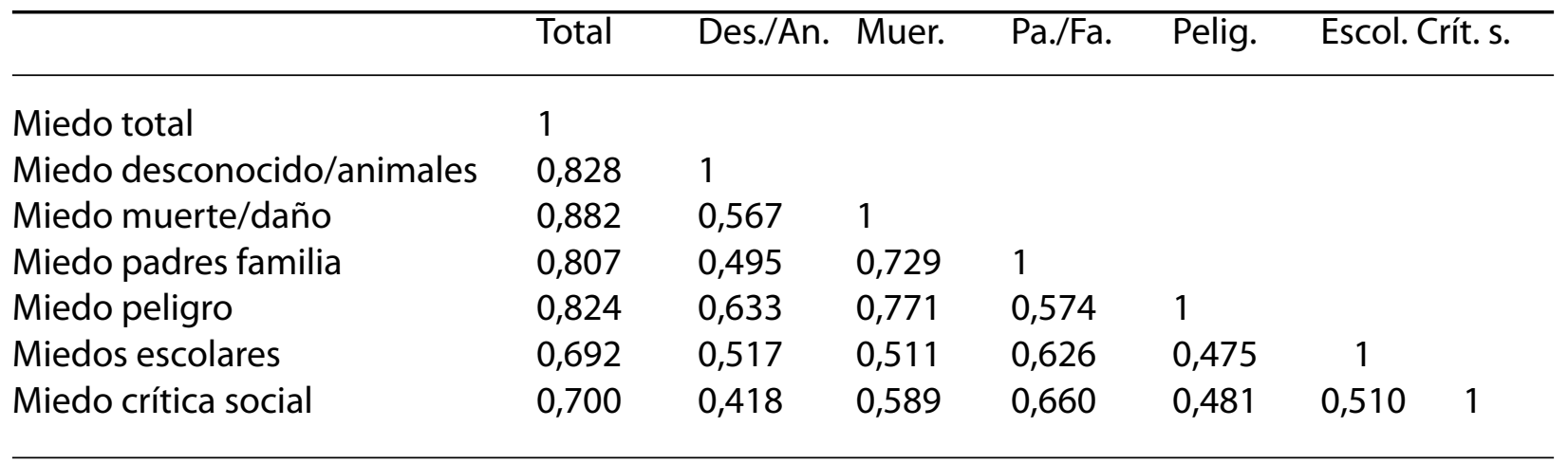

Nota: la correlación es significativa al nivel 0,01 en todos los casos. Total = Miedo total; Des./an. = miedo a lo desconocido y los animales; Muer. = Miedo a la muerte y al daño; $\mathrm{Pa} . / F a .=$ Miedo provocado por padres y familiares; Pelig. $=$ Miedo al peligro; Escol. $=$ Miedos provocados por diferentes fenómenos escolares; Crít. $\mathrm{s} .=$ Miedo ante la crítica social. 
Tabla 9

Correlaciones entre los factores en chicas

\begin{tabular}{lllllll}
\hline & Total & Muert. & Des./m. & Esc./Cr. & Anim. & Soled. \\
\hline Miedo Total & 1 & & & & & \\
Miedo Muerte/Daño & 0,862 & 1 & & & & \\
Miedo Desconocido/médicos & 0,810 & 0,488 & 1 & & & \\
Miedos Escolares/Crítica & 0,796 & 0,652 & 0,548 & 1 & & \\
Miedo Animales & 0,764 & 0,641 & 0,557 & 0,441 & 1 & \\
Miedo Soledad & 0,762 & 0,545 & 0,665 & 0,582 & 0,494 & 1 \\
\hline
\end{tabular}

Nota: La correlación es significativa al nivel 0,01 en todos los casos. Tot. = Miedo total; Muert. = Miedo a la muerte y al daño; Des. $/ \mathrm{m}$. = Miedo a lo desconocido y a los médicos; Esc./cr. = Miedo relacionado con elementos escolares y la crítica; Anim. = Miedo a los animales; Soled. = Miedo a la soledad.

al peligro y los más bajos $(0,418)$ encontrados entre el miedo a la crítica social el miedo a lo desconocido y a los animales.

Lo mismo se realizó entre las chicas, donde de nuevo, todas las correlaciones fueron significativas al nivel $p=0,01$ (Tabla 9). Las correlaciones más bajas entre los totales y los factores se dan entre el miedo total y el factor miedo a la soledad $(0,762)$. Las más altas se dan entre los totales y el factor de miedo a la muerte y al daño $(0,862)$. Lo mismo se repite considerando las intercorrelaciones de los factores entre sí. En este sentido, los niveles se mueven entre los más altos $(0,665)$ encontrados en las correlaciones entre el miedo a la soledad y el miedo a lo desconocido y a los médicos y los más bajos $(0,441)$ encontrados entre el miedo a los animales y los miedos escolares y a la crítica.

A continuación se comentan los resultados de la prueba ANOVA, con la intención de comprobar la existencia de diferencias significativas en función de la variable género. En este sentido, se consideró sólo el factor que coincide en ambos géneros (con los mismos ítems).
En el miedo a la muerte y al daño $(p<0,001)$, se obtuvieron diferencias significativas. En esta categoría las chicas evidencian niveles más altos de miedo que los chicos, tal y como indican sus medias (las chicas 2,14 y los varones 1,61).

Los niveles más altos de miedo entre las chicas se dan en el factor de miedo a la muerte y al daño (su media es de 2,14) y los más bajos en el miedo a lo desconocido y a los médicos $(0,98)$. Para los varones, los más altos se dan también en miedo a la muerte y al daño (1.61); sin embargo, los más bajos $(0,66)$ se dan en la categoría miedo a lo desconocido y a los animales.

\section{Discusión}

Se parte en el presente estudio con la idea de validar y comprobar las propiedades psicométricas de un instrumento para evaluar el miedo, baremado en una muestra adolescente por Ascensio et al. (2012) tomando como muestra una población infantojuvenil. Se aplicó el análisis por separado de varones y mujeres, intentando contemplar las diferencias en 
cuanto a los factores, considerando el género, siguiendo el estudio de Matesanz (2006). Los niveles de consistencia interna en ambos son prácticamente idénticos, lo mismo que con respecto al instrumento de referencia $(=0,961$ los varones $\mathrm{y}=0,955$ las chicas) y también elevados y similares a los obtenidos en el mismo para cada uno de los factores ( desde 0,934 hasta 0,700 en los varones y desde 0,918 hasta 0,716 entre las chicas). Esta realidad se muestra muy similar a la obtenida en el trabajo de Matesanz (2006). Respecto a la dimensionalidad del instrumento, se encontraron bastantes diferencias entre ambos géneros, algo que también ocurrió en el estudio mencionado (Matesanz, 2006). En el caso de las chicas se obtuvo el mismo número de factores (5) que en el instrumento de referencia (Asecnsio et al., 2012). Estos factores son miedo a la muerte y al daño (segundo factor en los chicos), miedo a lo desconocido y a los médicos (integra un factor que aparece en otra categoría en los varones. No obstante, los ítems relacionados con los médicos se distribuyen entre sus diferentes factores), miedos escolares (coincide con el quinto factor en los varones) y a la crítica (forma parte de otro factor en los varones, por lo que ambas categorías no se pueden equiparar), miedo a los animales (queda, junto con elementos relacionados con lo desconocido en los varones) y el miedo a la soledad (no coinciden los ítems con los encontrados para los chicos, repartiéndose los mismos por diferentes categorías). Para la muestra masculina el factor miedo a la muerte y al daño es prácticamente idéntico al encontrado en la muestra femenina. El resto (miedo a lo desconocido y a los animales, miedo a padres y familiares, miedo al peligro, miedos escolares y miedo a la crítica social) no coinciden o lo hacen sólo parcialmente, por lo que no se puede hablar de igualdad. Entre todos los factores suman una varianza explicada total del $48,308 \%$ en varones y de $46,829 \%$ en chicas (niveles similares entre sí y también con respecto al instrumento de referencia), quedando el cuestionario integrado, en ambos casos, por los 78 ítems que aparecen en esta versión. Se observa, por tanto, la importancia de aplicar el análisis por separado para ambos géneros, coincidiendo con el estudio de Matesanz (2006). Estos resultados, unidos a los referentes a las correlaciones (significativos al nivel $p=0,01$ en todos los casos) que se dan entre el miedo total y cada uno de los factores, de la misma manera que los obtenidos para las intercorrelaciones entre factores, apoyan el concepto jerárquico propuesto que parte de la existencia de un área unitaria (miedo) y una dimensionalidad múltiple (miedo ante estímulos más concretos). La validez convergente del instrumento esperaba obtener y obtuvo correlaciones positivas y estadísticamente significativas entre las dimensiones del instrumento en ambas muestras. Se encontraron diferencias significativas por género, tanto en los niveles totales como en el caso del miedo a la muerte y el daño. En ambos casos, la muestra femenina refleja niveles más altos. Esta realidad era algo que se esperaba, teniendo en cuenta los trabajos de Burnham et al. (2011), Miloyan et al. (2014) y Pulido y Herrera (2015). Esto puede relacionarse con la educación diferencial que reciben niños y niñas, correspondiente con los diferentes estilos de crianza de ambos 
(Méndez, Inglés, Hidalgo, García-Fernández, \& Quiles, 2003), lo que hace que las chicas sean más emocionales que los varones. Del mismo modo, para ambos grupos el factor de miedo que produce niveles más altos es el relacionado con la muerte y al daño. Resultados similares fueron encontrados por Burnham et al. (2011) y Pulido y Herrera (2015). Sin embargo, los niveles más bajos se dan en factores diferentes, ya que mientras que para las chicas se relacionan con el miedo a lo desconocido y los médicos, los varones temen menos a los animales y lo desconocido.

Finalmente indicar que el análisis de las propiedades psicométricas del cuestionario, en la población adolescente, permite ser optimistas en cuanto a la validez del cuestionario para medir los elementos pretendidos. Del mismo modo, puede ser un importante instrumento a emplear dentro del ámbito psicológico, social $y$, sobre todo, educativo en una población infantil y juvenil. En este sentido, el aprendizaje ha dado paso a una visión donde cada vez se incorporan más componentes socioafectivos (Quevedo-Blasco y Quevedo Blasco, 2014), siendo esencial la potenciación de diferentes programas de educación emocional, como elemento esencial del desarrollo educativo.

Con respecto a las diferencias entre chicos y chicas son evidentes, por lo que, siguiendo a Matesanz (2006) se destaca una mayor precisión y más valor predictivo, siendo el género un elemento discriminativo revelador de un rasgo femenino o masculino. Para evitar que la validez del instrumento se pueda ver negativamente afectada, lo más indicado es aplicar el análisis estadístico por separado para ambos géneros.

\section{Referencias}

Ascensio, M., Vila, M. G., Robles-García, R., Páez, F., Fresán, A., \& Vázquez, I. (2012). Estudio de traducción, adaptación y evaluación psicométrica del inventario de miedos FSSC-II en una muestra de estudiantes de educación media superior. Salud Mental, 35, 195-203.

Burnham, J., Hooper, L., \& Ogorchock, H. (2011). Differences in the fears of elementary school children in North and South America: A cross-cultural comparison. International Journal for the Advancement of Counselling, 33, 235-251. Recuperado de http://link.springer.com/article/10.1007\%2 Fs10447-011-9131-7

Codero, J. M., \& Manchón, C. (2014). Factores explicativos del rendimiento en educación primaria: un análisis a partir de TIMSS 2011. Estudios Sobre Educación, 27, 9-35.

Filella, G., Pérez, N., Agulló, M. J., \& Oriol, X. (2014). Resultados de la aplicación de un programa de educación emocional en Educación Primaria. Estudios Sobre Educación, 26, 125-147.

García-Fernández, J. M., Inglés, C. J., MartínezMonteagudo, M. C., Marzo, J. C., \& Estévez, E. (2011). Inventario de Ansiedad Escolar: validación en una muestra de estudiantes de Educación Secundaria. Psicothema, 23, 301-307.

Gómez-Ortiz, O., Casas, C., \& Ortega-Ruiz, R. (2016). Ansiedad social en la adolescencia: factores psicoevolutivos y de contexto familiar. Behavioral Psychology/Psicología Conductual, 24, 29-49.

Ford, B., Mauss, I., Troy, A., Smolen, A., \& Hankin, B. (2014). Emotion regulation moderates the risk associated with the 5-HTT gene and stress in children. Emotion, 14, 930 -939.

Kushnir, J., Gothelf, D., \& Sadeh, A. (2014). Nighttime fears of preschool children: A potential disposition marker for anxiety? Comprehensive Psychiatry, 55, 336-341.

Matesanz, A. (2006). Datos para la adaptación castellana de la Escala de Temores (FSS). Análisis y Modificación de Conducta, 32, 521551.

Méndez, F. X., Inglés, C. J., Hidalgo, M. D., García-Fernández, J. M., \& Quiles, M. J. (2003). 
Los miedos en la infancia y la adolescencia: un estudio descriptivo. Revista Electrónica de Motivación y Emoción, 6, 13-25. Recuperado de http://reme.uji.es

Mestre, J. M., Guil, R., Martínez-Cabañas, F., Larrán, C., \& González, G. (2011). Validación de una prueba para evaluar la capacidad de percibir, expresar y valorar emociones en niños de la etapa infantil. Revista Electrónica Interuniversitaria de Formación del Profesorado, 14(3), 37-54. Recuperado de web: http://www.aufop.com

Miloyan, B., Bulley, A., Pachana, N., \& Byrne, G. (2014). Social phobia symptoms across the adult lifespan. Journal of Affective Disorders, 168, 86-90.

Moreno-Rosset, C., Arnal-Remón, B., Antequera-Jurado, R., \& Ramírez-Uclés, I. (2016). Anxiety and psychological wellbeing in couples in transition to parenthood. Clínica y Salud, 27, 29-35. doi:10.1016/j.clysa.2016.01.004

Quevedo-Blasco, R., \& Quevedo Blasco, V. J. (2014). La equinoterapia como estrategia de intervención psicológica en adolescentes con problemas de conducta en el aula. Análisis y Modificación de Conducta, 40, 43-51. Recuperado de http://uhu.es/ publicaciones/ojs/index.php/amc/article/ view/2613/2444

Pardo, R., \& Calero, A. (2016). Dificultades en la intervención psicológica de un caso clínico complejo de ansiedad y obsesiones: la importancia del análisis funcional. Análisis y Modificación de Conducta, 41, 17-39. Recuperado de http://uhu.es/publicaciones/ ojs/ index.php/amc/article/view/2763

Pulido, F., \& Herrera, F. (2015). Miedo e inteligencia emocional en el contexto luricultural de Ceuta. Anuario de Psicología, 45, 249-263.

Roca-Sánchez, M. J., Álvarez-Pérez, Y., \& Peñate, W. (2014). Aceptabilidad de un tratamiento vía internet para la agorafobia por pacientes y terapeutas. Análisis y Modificación de Conducta, 40, 29-41. Recuperado de http:// uhu.es/publicaciones/ojs/index.php/amc/ article/view/2612/2441

Roth, G., Benita, M. Amrani, C., Shachar, B. Asoulin, H., Moed, A.,... Kanat-Maymon, Y.
(2014). Integration of negative emotional experience versus suppression: Addressing the question of adaptive functioning. Emotion, 14, 908-919. 

